Notre Dame Journal of Formal Logic

Volume 48, Number 1, 2007

\title{
A Note on Counterexamples to the Vaught Conjecture
}

\author{
Greg Hjorth
}

\begin{abstract}
If some infinitary sentence provides a counterexample to Vaught's Conjecture, then there is an infinitary sentence which also provides a counterexample but has no model of cardinality bigger than $\aleph_{1}$.
\end{abstract}

\section{Introduction}

The following theorem was shown in [2].

Theorem 1.1 There is a countable structure $\mathcal{M}$ with $S_{\infty}$ dividing $\operatorname{Aut}(\mathcal{M})$ and whose Scott sentence has models of size $\aleph_{1}$ but no higher.

This note makes the following corollary of that theorem explicit.

Corollary 1.2 If there is some $\sigma \in \mathscr{L}_{\omega_{1} \omega}$ providing a counterexample to Vaught's Conjecture for $\mathcal{L}_{\omega_{1} \omega}$, then there is some $\sigma^{\prime} \in \mathcal{L}_{\omega_{1} \omega}$ which again provides a counterexample and has no model of cardinality bigger than $\aleph_{1}$.

Harrington has shown that any counterexample to Vaught's Conjecture will have, necessarily uncountable, models with arbitrarily high Scott ranks below $\omega_{2}$.

\section{Proof}

For convenience, we work under $\neg \mathrm{CH}$. A similar argument works for the version of the Vaught Conjecture which asks for uncountably many nonisomorphic countable models without a perfect set of nonisomorphic countable models.

Received August 15, 2005; accepted November 8, 2005; printed February 26, 2007

2000 Mathematics Subject Classification: Primary, 03E15

Keywords: Vaught's conjecture, infinitary logic, Polish group

(c) 2007 University of Notre Dame 
Definition 2.1 $S_{\infty}$ is said to divide a Polish group $G$ if there is a closed subgroup $H<G$ and a continuous, onto, homomorphism

$$
\pi: H \rightarrow S_{\infty} .
$$

Notation 2.2 For $\mathcal{L}$ a countable language we let $X_{\mathscr{L}}$ be the space of all $\mathcal{L}$ structures with underlying set $\mathbb{N}$ and with the topology having basic open sets

$$
\{\mathcal{N}: \mathcal{N} \models \psi(\vec{a})\}
$$

for $\psi$ quantifier free and $\vec{a}$ a finite sequence from $\mathbb{N}$.

It is well known and easily checked that $X_{\mathcal{L}}$ is a Polish space in the indicated topology. Compare [1], §2.7.

Let $\mathcal{M}$ be as in the statement of the theorem and let $\sigma \in \mathcal{L}_{\omega_{1} \omega}$ have exactly $\aleph_{1}$ many nonisomorphic models. For convenience I will assume that $\mathcal{M}$ has $\mathbb{N}$ as its underlying set. Fix $H$ a closed subgroup of $\operatorname{Aut}(\mathcal{M})$ which maps continuously and homomorphically onto $S_{\infty}$.

In particular, we have a continuous action of $S_{\infty}$ on a Polish space $X$ with exactly $\aleph_{1}$ many orbits. This obviously can be lifted up to a continuous action of the Polish group $H$ with the same orbit structure, and then following [1], 2.3.5, we may lift the action of $H$ on $X$ up to an action of $\operatorname{Aut}(\mathcal{M})$ on a Polish space $Y \supset X$ such that, among other things, $Y$ has the same number of orbits under $\operatorname{Aut}(\mathcal{M})$ as $X$ does under $H$. That is to say, $Y / \operatorname{Aut}(\mathcal{M})$ has size $\aleph_{1}$.

Then by [1], 2.7.4, we can find a richer countable language $\mathscr{L}^{\prime} \supset \mathscr{L}$ such that the expansions of $\mathcal{M}$ to $\mathcal{L}^{\prime}, Y_{\mathcal{L}^{\prime}}^{\mathcal{M}}$, form a universal Polish $\operatorname{Aut}(\mathcal{M})$ space. In particular, there will be an injective and Borel $\operatorname{Aut}(\mathcal{M})$-map,

$$
\rho: Y \rightarrow Y_{L^{\prime}}^{\mathcal{M}}
$$

Since the injective images of Borel sets are Borel, there will be an invariant Borel set

$$
B \subset Y_{L^{\prime}}^{\mathcal{M}}
$$

which contains exactly $\aleph_{1}$ many orbits under the action of Aut $(\mathcal{M})$.

Now let $C$ be the set of all $\mathcal{N} \in X_{\mathscr{L}^{\prime}}$ such that there exists $\mathcal{N}^{\prime} \cong \mathcal{N}$ with $\mathcal{N}^{\prime} \in B$. Note that $C$ is then $\Delta_{1}^{1}$, and hence Borel, since, by invariance of $B$, we can equivalently describe $C$ as the set of $\mathcal{N} \in X_{\mathscr{L}^{\prime}}$ which satisfy $\varphi_{\mathcal{M}}$, the Scott sentence of $\mathcal{M}$, and for which any $\mathcal{N}^{\prime} \in Y_{\mathcal{L}^{\prime}}^{\mathcal{M}}$ isomorphic to $\mathcal{N}$ is in $B$.

Thus by [3], there is some $\sigma^{\prime} \in \mathcal{L}_{\omega_{1} \omega}^{\prime}$ whose models with underlying set $\mathbb{N}$ are exactly the elements of $C$. Since $\sigma^{\prime} \Rightarrow \varphi_{\mathcal{M}}, \sigma^{\prime}$ has no model of size $\aleph_{2}$. By construction, $\sigma^{\prime}$ has exactly $\aleph_{1}$ many nonisomorphic models.

\section{References}

[1] Becker, H., and A. S. Kechris, The Descriptive Set Theory of Polish Group Actions, vol. 232 of London Mathematical Society Lecture Note Series, Cambridge University Press, Cambridge, 1996. Zbl 0949.54052. MR 1425877. 50

[2] Hjorth, G., "Knight's model, its automorphism group, and characterizing the uncountable cardinals," Journal of Mathematical Logic, vol. 2 (2002), pp. 113-44. Zbl 1010.03036. MR 1900550. 49 
[3] Lopez-Escobar, E. G. K., "An interpolation theorem for denumerably long formulas," Fundamenta Mathematicae, vol. 57 (1965), pp. 253-72. Zbl 0137.00701. MR 0188059. 50

\section{MSB 6363}

UCLA

Los Angeles CA 90095-1555

greg@math.ucla.edu 